\title{
A NECESSARY AND SUFFICIENT CONDITION FOR A 3-MANIFOLD TO HAVE HEEGAARD GENUS ONE
}

\author{
JOEL HASS AND ABIGAIL THOMPSON
}

(Communicated by Irwin Kra)

\begin{abstract}
Let $M$ be a closed 3-manifold. R. H. Bing showed that $M$ is homeomorphic to $S^{3}$ if and only if every simple closed curve in $M$ can be isotoped to lie inside a 3-ball. We generalize this to show that there is a solid torus $T$ imbedded in $M$ such that every simple closed curve in $M$ can be isotoped to lie in $T$ if and only if $M$ has a genus one Heegaard splitting.
\end{abstract}

In [1], R. H. Bing gives a necessary and sufficient condition for a 3-manifold to be $S^{3}$ :

Theorem. Let $M^{3}$ be a closed 3-manifold. Every simple closed curve in $M^{3}$ can be isotoped to lie inside a 3-ball if and only if $M^{3}$ is homeomorphic to $S^{3}$.

This theorem is closed related to the Poincare conjecture; if we replace isotoped in the above statement by homotoped we obtain:

Poincaré Conjecture. Let $M^{3}$ be a closed 3-manifold. Every simple closed curve in $M^{3}$ can be homotoped to lie inside a 3-ball if and only if $M^{3}$ is homeomorphic to $S^{3}$.

There have been many generalizations of Bing's theorem (see [2], [5], [6], [7]), all of which show that one can weaken the hypotheses of the theorem in various ways and still conclude that the manifold is $S^{3}$. It is shown in [5] that if every knot in $M$ is contractible in a genus one handlebody then the manifold is $S^{3}$. In [7] it is shown that the same is true if every knot in $M$ is contractible in a genus two handlebody. We examine the situation where the assumption of contractibility in the handlebody is dropped. Any knot can be isotoped into some genus one handlebody, namely its regular neighborhood. We generalize Bing's theorem by considering what happens when every knot can be isotoped into a fixed genus one handlebody. Any two 3-balls in a manifold are isotopic so this gives a direct generalization of Bing's theorem. We obtain a necessary and sufficient condition for a manifold to have Heegaard genus one.

Received by the editors December 28, 1988.

1980 Mathematics Subject Classification (1985 Revision). Primary 57M25. 
Theorem 1. Let $M$ be a closed 3-manifold. There is a solid torus $T$ imbedded in $M$ such that every simple closed curve in $M$ can be isotoped to lie in $T$ if and only if $M$ has a genus one Heegaard splitting.

Remark. This implies that $M$ is homeomorphic to a Lens space, $S^{3}$, or $S^{1} \times$ $S^{2}$.

Theorem 1 suggests the following:

Conjecture. Let $M$ be a closed 3-manifold. There is a genus $g$ handlebody $H$ imbedded in $M$ such that every simple closed curve in $M$ can be isotoped to lie in $H$ if and only if $M$ has a genus $g$ Heegaard splitting.

A theorem due to R. Myers [7] guarantees the existence of certain special knots in any 3-manifold. We will need a generalization of this result.

Notation. If $X$ is a manifold, let $\dot{X}$ be the interior of $X$ and let $N(X)$ be a closed regular neighborhood of $X$.

Definiton. A knot $K$ in $M$ is simple if $M-\dot{N}(K)$ is irreducible and boundary irreducible and contains no properly imbedded nonboundary parallel incompressible annuli or tori.

Theorem 2 [7]. Every compact, orientable 3-manifold $M$ such that $\partial M$ contains no 2-spheres contains a simple knot $K$.

For our purposes we will only use that $M-\stackrel{\circ}{ }(K)$ is irreducible and contains no imbedded nonboundary parallel incompressible tori.

Definition. Two knots $K_{0}$ and $K_{1}$ in $M$ are equivalent if there exists an isotopy $h$ of $M$ such that $h\left(K_{1}\right)=K_{2}$. If $K_{1}$ and $K_{2}$ are not equivalent then they are distinct. Notice that distinct knots may have homeomorphic complements but knots with nonhomeomorphic complements are distinct.

Proposition 3. Every compact, orientable 3-manifold $M$ such that $\partial M$ contains no 2-spheres contains an infinite number of distinct simple knots.

Proof. In fact we show that $M$ contains an infinite number of simple knots with nonhomeomorphic complements.

We first give an outline of Myers' proof of Theorem 2:

Step 1. Construct a special handle decomposition of $M$ (see [7]).

One of the properties of such a decomposition is that every 0-handle meets exactly four 1 -handles. Label the 0 -handles $h_{1}, h_{2}, \ldots, h_{n}$. Let $B_{1}, \ldots, B_{n}$ be closed regular neighborhoods of the 0 -handles. Note that in this decomposition $n>2$.

Step 2. Into every $B_{i}, i=1, \ldots, n$, insert a copy $L_{i}$ of the 'true lovers' tangle', so that $\left(\bigcup L_{i}\right) \cup$ (cores of the 1-handles) forms a knot $K$ in $M$.

Step 3. Show that $K$ is simple.

The fact that one obtains a simple knot via this construction is independent of which special handle decomposition of $M$ one starts with. 
In order to construct an infinite number of distinct simple knots, we need the following:

Fact 1. Let $S_{i}=\partial B_{i}, i=1, \ldots, n$. The 4-punctured spheres $S_{i}-(\stackrel{\circ}{N}(K))$ properly imbedded in $M-\dot{N}(K)$ are incompressible and no two are parallel.

Proof. Suppose $S_{i}$ is compressible in $M-\stackrel{N}{N}(K)$. Let $D$ be a compressing disk. We can assume, by an innermost disk argument, that $D \cap\left(\bigcup S_{j}\right)=\partial D$. Since the tangle $L_{i}$ is prime, $D$ cannot be contained in $B_{i}-\stackrel{N}{N}\left(L_{i}\right)$, hence $S_{i}$ is compressible in $M-\stackrel{\circ}{N}\left(K \cup\left(\cup B_{j}\right), j=1, \ldots, n\right.$. This contradicts [7, Lemma 5.2], which states that the $S_{j}$ 's are incompressible in $M-\dot{N}\left(K \cup\left(\cup B_{j}\right)\right.$.

Suppose $S_{i}$ and $S_{j}$ are parallel in $M-\dot{N}(K)$. Then $M-\dot{N}(K)=\left(B_{i}-\right.$ $\stackrel{\circ}{N}(K)) \cup_{S_{i}}\left(S_{i} \times I\right) \cup_{S_{j}}\left(B_{j}-N(K)\right)$ and $n=2$. In a special handle decomposition we always have that $n>2$.

Fact 2. For every $h \in \mathbf{Z}_{+}$there exists a special handle decomposition of $M$ with more than $h$ 0-handles.

Proof. One can construct a special handle decomposition $H$ from any triangulation $T$ of $M$ with (the number of 0 -handles in $H$ ) $=($ the number of 3 simplices in the second barycentric subdivision of $T$ ). This can be chosen to be arbitrarily large.

We need also the following theorem, due to Haken [3] (as strengthened by Jaco-Shalen [4, Theorem III.24]):

Theorem 4. Let $M$ be a compact, orientable 3-manifold. There is an integer $n(M)$ such that if $\left\{F_{1}, \ldots, F_{k}\right\}$ is any collection of pairwise disjoint, incompressible surfaces properly imbedded in $M$, then either $k<n(M)$, some $F_{i}$ is a disk or annulus parallel into $\partial M$, or for some $i \neq j, F_{i}$ is parallel to $F_{j}$ in $M$.

Let $K_{0}$ be the simple knot obtained via Myers' construction. Let $M_{0}=$ $M-\stackrel{\circ}{N}\left(K_{0}\right)$. Using Fact 2, we find a special handle decomposition of $M$ with $h$ 0-handles, where $h>n\left(M_{0}\right)$. Let $K_{1}$ be the simple knot obtained via Myers' construction using this special handle decomposition. Then $M_{1}=M-\stackrel{\circ}{N}\left(K_{1}\right)$ contains $h$ incompressible, nonparallel surfaces, none of which is a disk or annulus. Hence by Theorem $4, M_{1}$ is not homeomorphic to $M_{0}$. Hence $K_{0}$ and $K_{1}$ are distinct. One can continue this process to obtain an infinite number of distinct simple knots.

Proof of Theorem 1. Let $K_{1}$ and $K_{2}$ be two distinct simple knots in $M$. Isotop $K_{1}$ to lie in $T$. Since $K_{1}$ is simple, $\partial T$ is either compressible or boundary parallel in $M-\stackrel{\circ}{N}\left(K_{1}\right)$.

Suppose $\partial T$ is compressible. Let $D$ be a compressing disk for $\partial T$. 
(i) Suppose $D \subset T$. Then $K_{1}$ lies inside a 3-ball $B_{1}=T-D$. Since $K_{1}$ is simple, $\partial B$ must bound a 3 -ball $B_{2}$ in $M-\dot{N}(K)$. Hence $M=B_{1} \cup_{\partial} B_{2}$, so $M=S^{3}$.

(ii) Suppose $D \subset(M-\dot{T})$. Let $S$ be the 2-sphere obtained by compressing $\partial T$ along $D$. Since $K_{1}$ is simple, $S$ must bound a ball in $M-\stackrel{\circ}{N}\left(K_{1}\right)$, hence $M-T$ is a solid torus $T^{\prime}$. So $M=T \cup_{\partial} T^{\prime}$; hence $M$ has a genus one Heegaard splitting.

So either $M$ has a genus one Heegaard splitting or $\partial T$ is boundary parallel in $M-\dot{N}\left(K_{1}\right)$. Note that this implies that $K_{1}$ is equivalent to the core of $T$. Repeating the argument using $K_{2}$, we can conclude that either $M$ has a genus one Heegaard splitting or $\partial T$ is boundary parallel in $M-\stackrel{N}{N}\left(K_{2}\right)$, and hence $K_{2}$ is equivalent to the core of $T$. Since $K_{1}$ and $K_{2}$ are distinct, $\partial T$ cannot be boundary parallel in both their complements, hence $M$ has a genus one Heegaard splitting. This concludes the proof of the first implication of Theorem 1. The converse follows by transversality, concluding the proof.

\section{REFERENCES}

1. R. H. Bing, Necessary and sufficient conditions that a 3-manifold be $S^{3}$, Ann. of Math. 68 (1958), 17-37.

2. C. McA. Gordon and J. M. Montesinos, Fibred knots and disks with clasps, M.S.R.I. preprint 13912-85, 1985.

3. W. Haken, Some results on surfaces in 3-manifolds, Studies in Modern Topology, Math. Assoc. Amer., distributed by Prentice Hall, 1968, 34-98.

4. W. Jaco, Lectures on three-manifold topology, C.B.M.S., Vol. 43, 1980.

5. D. R. McMillan, On homologically trivial 3-manifolds, Trans. Amer. Math. Soc. 98 (1961), 350-367.

6. R. Myers, Open book decompositions of 3-manifolds, Proc. Amer. Math. Soc. 72 (1978), 397402.

7. __ Simple knots in compact, orientable 3-manifolds, Trans. Amer. Math. Soc. 273 (1982), $75-91$. 